\title{
Topical formulations to prevent sexually transmitted infections: are we on track?
}

José A Fernández Romero*,1,2, Maria Gabriela Paglini ${ }^{3,4}$ \& Thomas M Zydowsky²

${ }^{1}$ Borough of Manhattan Community College, 199 Chambers Street, NY 10007, USA

${ }^{2}$ Center for Biomedical Research, Population Council, 1200 York Avenue, NY 10065, USA

${ }^{3}$ Instituto de Virología "Dr JM Vanella", Facultad de Ciencias Médicas, Universidad Nacional de Córdoba, Córdoba CP 5016 ,

Argentina

${ }^{4}$ Instituto de Investigación Médica Mercedes y Martín Ferreyra, INIMEC-CONICET-Universidad Nacional de Córdoba, Córdoba CP

5016, Argentina

*Author for correspondence: jfernandezromero@bmcc.cuny.edu

\section{“A potential solution that has been gaining momentum is the development of novel noncondom multipurpose prevention technologies (MPTs) that prevent unintended pregnancy and HIV/STIs simultaneously."}

First draft submitted: 15 June 2019; Accepted for publication: 8 July 2019; Published online: 22 August 2019

It is estimated that more than one million people acquire a sexually transmitted infection (STI) every day. 4 years ago, we started a Commentary paper in this journal with the same statement [1]. The numbers have not changed; if anything, based on recent estimates, they may have actually increased [2]. Annually, there are an estimated 357 million new bacterial STIs caused by Chlamydia trachomatis, Neisseria gonorrhoeae, Treponema pallidum and Trichomonas vaginalis. The viral noncurable STIs also show high prevalence with more than 500 million people having genital infections with herpes simplex virus (HSV), more than 290 million women infected with HPV and more than 37 million people living with HIV globally [3].

Many of these STIs could be asymptomatic and only systematic testing for the presence of these infections may allow treatment to manage or cure the disease. Additionally, infections by STIs like HSV, T. vaginalis and T. pallidum can increase the risk of HIV acquisition. Congenital syphilis after vertical transmission of T. pallidum can result in stillbirths and newborn death. STIs can also result in a carcinogenic process, pelvic inflammatory disease and/or infertility. Lately, the rapid spread of antibiotic resistance in $N$. gonorrhoeae infections has added one more obstacle to control STIs worldwide [3].

If the numbers above do not scare you because you practice safe sex and you have never experienced an STI, indirectly, they are still affecting you. A study looked at the total lifetime direct medical cost of STIs diagnosed and treated in 2008 in the USA, estimating a total cost of US $\$ 15.6$ (range: US\$11.0-20.6) billion. Viral noncurable STIs accounted for approximately $92 \%$ of total cost, and chlamydia was the most-costly nonviral infection [4].

\section{What is available to prevent STIs?}

Abstinence or having a monogamous partner who is not infected with an STI can stop the spread of STIs. However, abstinence is not an option in a sexually active population. Male and female condoms are still the only medical devices available to reduce the risk of acquiring an STI while also preventing unintended pregnancy. The female condom, now called the single-use internal condom, has been investigated as a female-controlled method that can protect against both pregnancy and STIs [5]. The US FDA recently approved new rules regarding the device's name and some other regulations. These changes have made clear the approval for use in the vaginal and anal tract with the same regulatory classification as the male condom [6].

Although condoms have the potential to be an excellent self-initiated method for STI prevention, condom-based prevention strategies have not been effective at stopping the global increase in STIs prevalence, indicating that alternative prevention strategies need to be investigated. This is in part needed because typical condom use can be inconsistent, and women are not always able to ensure their partner uses them. This does not rule out that improving sexual education could help to increase the use of these important prevention tools.

Future Medicine 
Globally, young women, men who have sex with men and sex workers are some of the high-risk populations in desperate need for tools that could alleviate the current lack of choices for STI prevention. A potential solution that has been gaining momentum is the development of novel noncondom multipurpose prevention technologies (MPTs) that prevent unintended pregnancy and HIV/STIs simultaneously [7]. Some of the strategies under development may also provide a woman-initiated product that could reduce the need for partner negotiation.

\section{What does the MPT pipeline look like?}

Several promising MPT formulations/devices are in the late preclinical development/clinical trials. Amphora ${ }^{\circledR}$ contraceptive vaginal gel is a multipurpose vaginal $\mathrm{pH}$ regulator developed by Evofem Biosciences, Inc. The gel regulates vaginal $\mathrm{pH}$ within the normal range of 3.5-4.5, maintaining an acidic environment that is not tolerated by sperm and potentially many pathogens like C. trachomatis and $N$. gonorrhoeae. AMPOWER, a Phase III contraceptive efficacy trial, indicated typical use efficacy of $86 \%$ and perfect use efficacy of 99\% [8]. These results are comparable to the most commonly used forms of hormonal contraception (i.e., pills). A double-blinded placebo-controlled Phase IIb clinical trial (AMPREVENCE) is currently evaluating Amphora for the prevention of urogenital $C$. trachomatis and $N$. gonorrhoeae in women [9]. The company is also preparing for trials for prevention of recurrent bacterial vaginosis.

PC-1005 (MIV-150, zinc acetate and carrageenan [CG]) is a broad-spectrum, dual compartment formulation with proven antiviral activity against HIV-1, HSV-2 and HPV in preclinical animal models. PC-1005 is the first dual compartment MPT formulation that completed clinical trials in both the vaginal and rectal compartments. In a Phase I trial (PC-558), PC-1005 was found to be safe and well tolerated after vaginal application, once daily for 14 days [10]. Low systemic levels of MIV-150 were observed while plasma zinc levels were unchanged. Cervicovaginal lavages, collected from the participants showed anti-HIV and anti-HPV activities. MTN-037 is an escalating dose study to access the safety, pharmacokinetics and pharmacodynamics of rectally applied PC-1005. The data analysis is ongoing.

A broad spectrum MPT gel (HIV, HSV-2 and HPV in preclinical animal models) containing the antiviral agents griffithsin (GRFT) and CG was recently tested in a first-in-human trial [11]. The vaginal formulation was used once daily for 14 days. There were no serious adverse events related to the use of the vaginal formulation that was also well-tolerated. The GRFT/CG combination has also been delivered from vaginal rings and fast dissolving inserts. The fast dissolving inserts show good stability under accelerated temperature and humidity conditions [12]. Additionally, this MPT candidate significantly protected animals from SHIV, HSV-2 and HPV infections [13]. The presence of CG in the formulation provides an active pharmaceutical ingredient against HPV. An ongoing Phase IIb trial (CATCH study) is evaluating the efficacy of a carrageenan-based lubricant gel in reducing the risk of HPV infections in women. The interim analysis suggests that the gel containing CG can reduce the risk of HPV infections in women [14]. A rectal formulation containing GRFT is being evaluated by scientists at the University of Louisville in a Phase I clinical trial [15].

A tenofovir-based hyposmolar enema for rectal use has been designed as a behaviorally congruent strategy to prevent HIV and HSV-2 acquisition during receptive anal intercourse. This strategy takes advantage of a practice (enema use) that could fit the lifestyle of people engaging in high-risk receptive anal intercourse. Preclinical studies in mice and macaque show promising results and support the further development of this formulation in clinical trials [16]. A long-acting (3 months) intravaginal ring that releases the contraceptive agent levonorgestrel in addition to tenofovir is also in development [17]. The ring may prevent not only HIV and HSV, but also unintended pregnancy. CONRAD, the organization that developed the ring has ongoing trials in collaboration with the Microbicides Trial Network (MTN) to measure safety, acceptability and adherence of this promising MPT.

Topical application of monoclonal antibodies is another potential MPT strategy. A Phase I clinical trial is currently evaluating the safety of HIV and HSV antibodies formulated in a vaginal film (MB66) [18]. Antisperm antibodies could be delivered topically to promote sperm agglutination and mucus trapping, which could prevent pregnancy. Ultimately, a contraceptive MPT product that combines antibodies against sperm, HIV and HSV could be formulated in vaginal films and intravaginal rings. Antibodies could be delivered via intramuscular injections, since antibodies have been genetically engineered to achieve systemic half-lives of up to 3 months [18].

Something to keep in consideration when developing MPTs is to understand the target market of these products. Recent studies (TRIO and Quatro trials) explored the preferences of African women by asking their participants for feedback on different potential formulations/devices (oral tablets, vaginal rings, injectables, vaginal films, vaginal 
inserts and vaginal gels) $[19,20]$. The results indicated no one product was preferred by all participants, making the case for multiple MPT options and giving room for the development of many of the MPTs discussed above.

\section{How can we further advance the development of novel STI prevention technologies?}

We first need to acknowledge that no single design or delivery approach will meet the needs of those individuals who are at higher risk of contracting STIs. Individuals want choices, and their choices might change over the lifetime. Unfortunately, the current funding scenario does not recognize the need for multiple types of products, focused to a large extent on systemic drug delivery for sustained protection against HIV primarily. The scientific community working on MPTs development recognize that $\mathrm{R} \& \mathrm{D}$ for MPTs is expensive, lengthy and a single funding agency will not be able to support several viable candidates. It is important that governmental funding agencies, in conjunction with the private sector, collaborate and leverage investments in promising MPT products [21]. There is also a need for a more robust pipeline to guarantee the continuity in the development of better MPT candidates. A more robust pipeline will not be possible without solid funding opportunities for a broad range of products, especially user-initiated products.

\section{Financial \& competing interests disclosure}

The authors have no relevant affiliations or financial involvement with any organization or entity with a financial interest in or financial conflict with the subject matter or materials discussed in the manuscript. This includes employment, consultancies, honoraria, stock ownership or options, expert testimony, grants or patents received or pending, or royalties.

No writing assistance was utilized in the production of this manuscript.

\section{References}

1. Fernandez-Romero JA. Not just HIV: preventing other viral sexually transmitted infections. Future Virol. 10(11), 1191-1194 (2015).

2. Ramchandani MS, Golden MR. Confronting rising STIs in the era of PrEP and treatment as prevention. Curr. HIV/AIDS Rep. 16(3), 244-256 (2019).

3. WHO. Fact sheet on sexually transmitted infections (2019). www.who.int/en/news-room/fact-sheets/detail/sexually-transmitted-infections-(stis)

4. Owusu-Edusei K Jr, Chesson HW, Gift TL et al. The estimated direct medical cost of selected sexually transmitted infections in the United States, 2008. Sex. Transm. Dis. 40(3), 197-201 (2013).

5. Chen BA, Blithe DL, Muraguri GR et al. Acceptability of the woman's condom in a Phase III multicenter open-label study. Contraception 99(6), 357-362 (2019).

6. Kempner M. 'Female condom' gets a genderless rebrand from FDA. www.thebodypro.com/article/female-condom-gets-a-genderless-rebrand-from-fda

7. Fernandez-Romero JA, Deal C, Herold BC et al. Multipurpose prevention technologies: the future of HIV and STI protection. Trends Microbiol. 23(7), 429-436 (2015).

8. Evofem Biosciences announces positive top-line results of Phase III study evaluating Amphora for hormone-free birth control 2019. https://evofem.investorroom.com/2018-12-17-Evofem-Biosciences-Announces-Positive-Top-Line-Results-of-Phase-3-Study-Evaluat ing-Amphora-for-Hormone-Free-Birth-Control

9. Evofem Biosciences announces last patient enrolled for AMPREVENCE, the Phase IIb sexually transmitted infections trial of Amphora 2019. https://evofem.investorroom.com/2019-04-23-Evofem-Biosciences-Announces-Last-Patient-Enrolled-for-AMPREVENCE-the -Phase-2b-Sexually-Transmitted-Infections-Trial-of-Amphora

10. Friedland BA, Hoesley CJ, Plagianos M et al. First-in-human trial of MIV-150 and Zinc Acetate coformulated in a carrageenan gel: safety, pharmacokinetics, acceptability, adherence, and pharmacodynamics. J. Acquir. Immune Defic. Syndr. 73(5),489-496 (2016).

11. Population council research examines new prevention approaches for most-at-risk at HIVR4P 2018. www.popcouncil.org/news/population-council-research-examines-new-prevention-approaches-for-most-at

12. Lal M, Lai M, Ugaonkar S et al. Development of a vaginal fast-dissolving insert combining griffithsin and carrageenan for potential use against sexually transmitted infections. J. Pharm. Sci. 107(10), 2601-2610 (2018).

13. Derby N, Lal M, Aravantinou M et al. Griffithsin carrageenan fast dissolving inserts prevent SHIV HSV-2 and HPV infections in vivo. Nat. Commun. 9(1), 3881 (2018).

14. Magnan S, Tota JE, El-Zein M et al. Efficacy of a carrageenan gel against transmission of cervical HPV (CATCH): interim analysis of a randomized, double-blind, placebo-controlled, Phase IIB trial. Clin. Microbiol. Infect. 25(2), 210-216 (2019).

15. Girard L, Birse K, Holm et al. Impact of the griffithsin anti-HIV microbicide and placebo gels on the rectal mucosal proteome and microbiome in non-human primates. Sci. Rep. 8(1), 8059 (2018). 
16. Hoang T, Date AA, Ortiz JO et al. Development of rectal enema as microbicide (DREAM): preclinical progressive selection of a tenofovir prodrug enema. Eur. J. Pharm. Biopharm. 138, 23-29 (2019).

17. Thurman AR, Schwartz JL, Ravel J et al. Vaginal microbiota and mucosal pharmacokinetics of tenofovir in healthy women using tenofovir and tenofovir/levonorgestrel vaginal rings. PLoS ONE 14(5), e0217229 (2019).

18. Whaley K. Monoclonal antibodies as future MPT products. www.theimpt.org/blog-impt/item/monoclonal-antibodies-as-future-mpt-products

19. Shapley-Quinn MK, Manenzhe KN, Agot K et al. "We are not the same": African women's view of multipurpose prevention products in the TRIO clinical study. Int. J. Womens Health 11, 97-107 (2019).

20. Montgomery ET, Beksinska M, Mgodi N et al. End-user preference for and choice of four vaginally delivered HIV prevention methods among young women in South Africa and Zimbabwe: the Quatro Clinical Crossover Study. J. Int. AIDS Soc. 22(5), e25283 (2019).

21. Critical actions to achieve the value potential of MPTs for prevention of HIV, other STIs, and unintended pregnancies among young women. Presented at: International AIDS Conference. Amsterdam, The Netherlands, July 2018. www.theimpt.org/documents/reports/MPT-WorkshopSummaryReport_AIDS2018.pdf 